\title{
3-D Imaging using Row--Column-Addressed 2-D Arrays with a Diverging Lens
}

Bouzari, Hamed; Engholm, Mathias; Stuart, Matthias Bo; Nikolov, Svetoslav Ivanov; Thomsen, Erik Vilain; Jensen, Jørgen Arendt

\section{Published in:}

Proceedings of 2016 IEEE International Ultrasonics Symposium

Link to article, DOI:

10.1109/ULTSYM.2016.7728394

Publication date:

2016

Document Version

Peer reviewed version

Link back to DTU Orbit

Citation (APA):

Bouzari, H., Engholm, M., Stuart, M. B., Nikolov, S. I., Thomsen, E. V., \& Jensen, J. A. (2016). 3-D Imaging using Row--Column-Addressed 2-D Arrays with a Diverging Lens. In Proceedings of 2016 IEEE International Ultrasonics Symposium IEEE. I E E E International Ultrasonics Symposium. Proceedings Vol. 2016 https://doi.org/10.1109/ULTSYM.2016.7728394

\section{General rights}

Copyright and moral rights for the publications made accessible in the public portal are retained by the authors and/or other copyright owners and it is a condition of accessing publications that users recognise and abide by the legal requirements associated with these rights.

- Users may download and print one copy of any publication from the public portal for the purpose of private study or research.

- You may not further distribute the material or use it for any profit-making activity or commercial gain

- You may freely distribute the URL identifying the publication in the public portal 


\title{
3-D Imaging using Row-Column-Addressed 2-D Arrays with a Diverging Lens
}

\author{
Hamed Bouzari*, Mathias Engholm ${ }^{\dagger}$, Matthias Bo Stuart*, \\ Svetoslav Ivanov Nikolov ${ }^{\ddagger}$, Erik Vilain Thomsen ${ }^{\dagger}$, and Jørgen Arendt Jensen* \\ * Center for Fast Ultrasound Imaging, Dept. of Elec. Eng., Bldg. 349, Technical University of Denmark, \\ 2800 Kgs. Lyngby, Denmark \\ $\dagger$ Dept. of Micro- and Nanotechnology, Technical University of Denmark, 2800 Kgs. Lyngby, Denmark \\ $\ddagger$ BK Ultrasound ApS, Herlev, Denmark
}

\begin{abstract}
It has been shown that row-column-addressed (RCA) 2-D arrays can be an inexpensive alternative to fully addressed 2-D arrays. Generally imaging with an RCA 2-D array is limited to its forward-looking volume region. Constructing a double-curved RCA 2-D array or applying a diverging lens over the flat RCA 2-D array, can extend the imaging field-of-view (FOV) to a curvilinear volume without increasing the aperture size, which is necessary for applications such as abdominal and cardiac imaging. Extended FOV and low channel count of doublecurved RCA 2-D arrays make it possible to have 3-D imaging with equipment in the price range of conventional 2-D imaging. This study proposes a delay-and-sum (DAS) beamformation scheme specific to double-curved RCA 2-D arrays and validates its focusing ability based on simulations. A synthetic aperture imaging (SAI) sequence with single element transmissions at a time, is designed for imaging down to $14 \mathrm{~cm}$ at a volume rate of $88 \mathrm{~Hz}$. The curvilinear imaging performance of a $\lambda / 2$-pitch $3 \mathrm{MHz} 62+62$ RCA 2-D array is investigated as a function of depth, using a diverging lens with f-number of $\mathbf{- 1}$. The results of this study demonstrate that the proposed beamforming approach is accurate for achieving correct time-of-flight calculations, and hence avoids geometrical distortions.
\end{abstract}

\section{INTRODUCTION}

An $N \times N$ element 2-D array can be operated utilizing only $2 N$ connections, when a row-column or cross-electrode addressing scheme is used [1]-[3]. This is contrary to the $N^{2}$ connections needed, when conventionally addressing the elements. In general, a row-column-addressed (RCA) array is a 2-D matrix array, which is addressed via its row- and column indices. Effectively, this makes two 1-D arrays arranged orthogonal to each other. As an example, a 256+256 RCA array will have 512 elements. A 2-D matrix array of equivalent size would have 65,536 elements, over a factor of 7 more than the current state-of-the-art X6-1 PureWave xMATRIX probe from Phillips (Eindhoven, Netherlands) that has 9212 elements [4]. This exhibits the potential of having very large RCA 2-D arrays with low channel count and real-time capabilities.

It has been demonstrated in several studies [1]-[3] that rowcolumn technology is a realistic alternative to the state-of-theart matrix probes, especially as a low-cost alternative. However, one major issue with the RCA arrays is that they can only emit acoustic energy directly below the array and in a cross-shape to the sides. For applications such as abdominal imaging, it is relevant to have a probe with a large aperture capable of phased array imaging. True volumetric phased array imaging is possible with RCA arrays, provided that the array is double curved to spread the energy during transmit [1]. However, manufacturing curved transducer elements is challenging for both capacitive micromachined ultrasonic transducer (CMUT) and piezoelectric transducer (PZT) technologies. Another approach to spread the acoustic energy is by using a double curved diverging acoustic lens on top of the RCA array [5]. Using a lens makes it easier to fabricate curved transducers, as it is not needed to manufacture curved elements, and also making a lens is a well-tested technology. An in-depth study of the possibilities of this approach is therefore the main goal of this study.

It is investigated whether curvilinear volumetric imaging is possible with an RCA array equipped with a diverging lens. A dedicated beamformer is developed and the performance is evaluated using Field II [6], [7] simulations. The quality assessments of the $\mathrm{B}$-mode images, i.e., spatial resolution and contrast resolution, are carried out on the simulated data using SAI technique. The remainder of the paper is organized as follows: The current limitations with flat RCA arrays and different approaches to disperse the acoustic energy are described in Section II. Section III presents a DAS beamformer for a double curved RCA array. In section IV, a detailed overview of the simulation setup is presented. Section V explains the simulation results and the final section concludes the paper.

\section{CURVED RCA 2-D ARRAYS}

To spread the acoustic energy of a line-element, the element has to be curved to generate a diverging wave. However, manufacturing double curved transducers is very challenging. Alternatively, the defocusing of the waves can be made by using a fixed electronic delay profile along each flat line-element to generate a diverging wavefront. This can be seen as using micro-beamforming with a fixed first stage. Another simpler approach to spread the acoustic energy is by using a double curved diverging acoustic lens on top of the RCA array [5]. A concave diverging lens can be designed with a material having a lower speed of sound compared to the human tissue, so that it has higher thickness around the corners and the sides of the array, and less thickness close to center of the array. 
Alternatively, a convex diverging lens can be made from a material with a higher speed of sound compared to the human tissue, which is preferred for a better contact surface. A flat diverging lens can also be made by using a combination of two different materials, one with higher and other one with lower speed of sound compared to the human tissue, which results in a flat surface for good contact.

Note that, for the same aperture size, lower lens $f_{\#}$ values for the lens corresponds to larger thicknesses of the lens, and therefore the attenuation becomes higher through the lens material. Thus, there is a trade-off between field-of-view (FOV) and attenuation. The $f_{\# \text { is defined as the ratio between the focal }}$ distance to the lens diameter. For example the delay profile can be in a range of $0 \mu$ s to $3.5 \mu$ s for a lens with $f_{\#}=-0.7$ and a speed of sound of $1400 \mathrm{~m} / \mathrm{s}$, which corresponds to a thickness range of $0 \mathrm{~mm}$ to $5 \mathrm{~mm}$. A suitable material for a lens could be Sylgard 160 (PDMS) with a density of $1580 \mathrm{~kg} / \mathrm{m}^{3}$ and a speed of sound of $950 \mathrm{~m} / \mathrm{s}$ and attenuation of $0.4 f^{1.4} \mathrm{~dB} / \mathrm{mm}$, where $f$ is the oprating frequency in $\mathrm{MHz}$. Therefore, for an operating frequency of $3 \mathrm{MHz}$ the maximum attenuation is $6.14 \mathrm{~dB}$ at the largest thickness [8]. This might be compensated by doubling the amplitude of the excitation pulse.

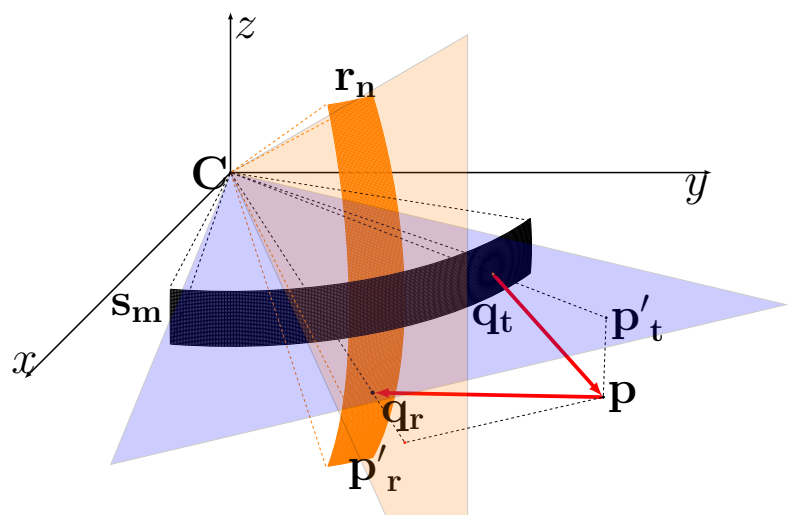

Fig. 1. The time-of-flight of a wavefront is given by the shortest distance from the source $\mathbf{s}_{m}$ to the point being focused $\mathbf{p}$ and back to the receiving element $\mathbf{r}_{n}$, divided by the speed of sound. The points $\mathbf{q}_{t}$ and $\mathbf{q}_{r}$ are the closest contact points on the transmit and receive elements $\mathbf{s}_{m}$ and $\mathbf{r}_{n}$ to the point $\mathbf{p}$.

\section{DAS BEAMFORMING WITH CURVED RCA 2-D ARRAYS}

The time-of-flight $(\mathrm{ToF})$ of a wavefront is given by the shortest distance from the arc source $\mathbf{s}_{m}$ to the point being focused $\mathbf{p}$, and back to the receiving element $\mathbf{r}_{n}$, divided by the speed of sound. Using the notations from Fig. 1 this can be written as:

$$
\operatorname{ToF}_{m}(n, \mathbf{p})=\frac{d\left(\mathbf{s}_{m}, \mathbf{p}\right)+d\left(\mathbf{r}_{n}, \mathbf{p}\right)}{c},
$$

where $c$ is the speed of sound in the medium, $n$ is an index from 1 to the number of receive line elements $N$, and $m$ is the emission index. The function $d(.,$.$) calculates the shortest$ distance between an arc and a point in space, which will be defined in the remeinder of this section.

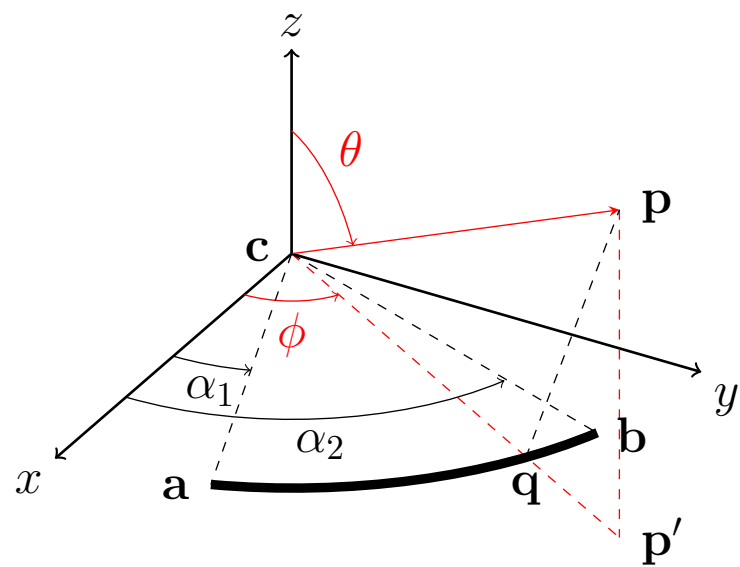

Fig. 2. Distance between a point $\mathbf{p}$ and an $\operatorname{arc} \overparen{\mathbf{a b}}$ is calculated using (4).

The arc segment from point $\mathbf{a}$ to point $\mathbf{b}$ with center $\mathbf{c}$ is termed $\mathbf{a b}$ assuming the center at origin. This is illustrated in Fig. 2. The projection of point $\mathbf{p}$ onto the plane passing through the arc ab and its center $\mathbf{c}$ is termed $\mathbf{p}^{\prime}$ and is determined by the usual equation for projection. To determine if the vector $\mathbf{c p}^{\prime}$ is in between vector $\mathbf{c a}$ and vector $\mathbf{c b}$, we define the normalized cross products $\hat{l}_{a}$ and $\hat{l}_{b}$ as

$$
\hat{l}_{a}=\frac{\mathbf{c p}^{\prime} \times \mathbf{c a}}{\left\|\mathbf{c} \mathbf{p}^{\prime}\right\|\|\mathbf{c a}\|}, \quad \hat{l}_{a}=\frac{\mathbf{c p}^{\prime} \times \mathbf{c b}}{\left\|\mathbf{c} \mathbf{p}^{\prime}\right\|\|\mathbf{c b}\|} .
$$

Depending on the location of the point $\mathbf{p}$, vectors $\hat{l}_{a}$ and $\hat{l}_{b}$ can be either $\hat{j}$ or $-\hat{j}$, where $\hat{j}$ is the unit vector of the $z$-axis. $\hat{l}_{a}$ and $\hat{l}_{b}$ have different signs, when $\alpha_{1} \leq \phi \leq \alpha_{2}$, and they have the same sign, when $\alpha_{2} \leq \phi$ or $\phi \leq \alpha_{1}$. Here $\alpha_{1}, \alpha_{2}$, and $\phi$ are the angles between the $x$-axis and the vectors $\mathbf{c a}, \mathbf{c b}$, and $\mathbf{c p}$, respectively, as shown in Fig. 2.

When $\hat{l}_{a}$ and $\hat{l}_{b}$ have different signs, i.e. $\hat{l}_{a}=\hat{j}$ and $\hat{l}_{b}=-\hat{j}$, or $\hat{l}_{a}=-\hat{j}$ and $\hat{l}_{b}=\hat{j}$, the standard formula for the distance between an arc and a point can be used:

$$
d=\sqrt{\left\|\mathbf{p p}^{\prime}\right\|^{2}+\left(\left\|\mathbf{c} \mathbf{p}^{\prime}\right\|-R\right)^{2}},
$$

where $R$ is the curvature of the arc and equal to $\|\mathbf{c a}\|$ or $\|\mathbf{c b}\|$.

When $\hat{l}_{a}$ and $\hat{l}_{b}$ have the same signs, i.e. $\hat{l}_{a}=\hat{j}$ and $\hat{l}_{b}=\hat{j}$, or $\hat{l}_{a}=-\hat{j}$ and $\hat{l}_{b}=-\hat{j}$, the shortest distance from the arc segment to the point is the distance from the closest end of the arc segment (a or $\mathbf{b})$ to the point $\mathbf{p}$. The following therefore determines the minimum distance between the point $\mathbf{p}$ and the arc segment ab:

$$
\begin{aligned}
& d(\widehat{\mathbf{a b}}, \mathbf{p})= \\
& \begin{cases}\sqrt{\left\|\mathbf{p p}^{\prime}\right\|^{2}+\left(\left\|\mathbf{c p}^{\prime}\right\|-R\right)^{2}} & \text { if } \begin{array}{l}
\hat{l}_{b}=-\hat{j} \text { and } \hat{l}_{a}=\hat{j} \\
\hat{l}_{b}=\hat{j} \text { and } \hat{l}_{a}=-\hat{j}
\end{array} \\
\|\mathbf{a p}\| & \text { if } \hat{l}_{b}=-\hat{j} \text { and } \hat{l}_{a}=-\hat{j} \\
\|\mathbf{b p}\| & \text { if } \hat{l_{b}}=\hat{j} \text { and } \hat{l}_{b}=\hat{j} .\end{cases}
\end{aligned}
$$


Using (4), the distances $d\left(\widetilde{\mathbf{s}_{m}}, \mathbf{p}\right)$ and $d\left(\overparen{\mathbf{r}_{n}}, \mathbf{p}\right)$ can now be determined. The focused signal at point $\mathbf{p}$ is calculated by summing all receive signals at the time instances given by (1):

$$
z_{m}(\mathbf{p})=\sum_{n=1}^{N} a_{\mathrm{elec}}(n, \mathbf{p}) y_{m, n}\left(\operatorname{ToF}_{m}(n, \mathbf{p})\right),
$$

where $N$ is the number of receive elements, $a_{\text {elec }}$ is the electronic receive apodization, and $y_{m, n}(t)$ is the measured signal from emission $m$ on the receive element $n$ at time $t$.

\section{Simulation And Measurement Setup}

In this work, Field II [6], [7] is used for all simulations. The simulated receive signals are beamformed using a MATLAB (MathWorks Inc., Massachusetts, USA) implemented the proposed DAS beamformer for curved RCA arrays. The simulation parameters of a RCA $62+62$ element 2-D array are shown in Table I. The receive array is rotated $90^{\circ}$ with respect to the transmit array. Field II is set up to use lines to describe the apertures and each line-element is divided into square mathematical sub-elements with a side length of $\lambda / 4$. To remove the otherwise apparent secondary echoes originating from the either ends of arc shaped elements, two roll-off apodization regions are placed at both ends of each element [9]. The length of each apodization region was equal to 15 times the pitch of the array. Each mathematical sub-element in both transmit and receive arrays is delayed according to the lens delay profile.

Table I

TRANSDUCER AND SIMULATION PARAMETERS.

\begin{tabular}{lccc}
\hline Parameter name & Notation & Value & Unit \\
\hline Number of elements & - & $62+62$ & - \\
Center frequency & $f_{0}$ & 3.0 & $\mathrm{MHz}$ \\
Speed of sound & $c$ & 1480 & $\mathrm{~m} / \mathrm{s}$ \\
Wave length & $\lambda$ & 493.3 & $\mu \mathrm{m}$ \\
Array pitch $-x$ & $d_{\mathrm{x}}$ & $\lambda / 2=246.6$ & $\mu \mathrm{m}$ \\
Array pitch $-y$ & $d_{\mathrm{y}}$ & $\lambda / 2=246.6$ & $\mu \mathrm{m}$ \\
Sampling frequency & $f_{s}$ & 120 & $\mathrm{MHz}$ \\
Emission pulse & - & 2-cycles, Hann-weighted & - \\
Lens focal ratio & $f_{\#}$ & -1 & - \\
\hline
\end{tabular}

\section{RESUlts AND Discussion}

In Fig. 3 the pulse-echo energy as a function of lateral position for different lens $f_{\#}$ is illustrated. For the flat array, the pulse-echo energy drops by moving away from the forward looking region of the array. This drop of the energy is due to the diffraction of the sound waves. At around $20 \mathrm{~mm}$ lateral position the pulse-echo energy drops by $40 \mathrm{~dB}$, when no lens is used. However, by using a diverging acoustic lens on top of an RCA 2-D array a larger FOV can be attained. The FOV can be adjusted by using different $f_{\#}$ values for the lens. The main advantage of using a diverging lens or designing a curved 2-D array, is to disperse the transmit and receive fields, so that they overlap in a larger area. By using a diverging lens with $f_{\#}=-1$, the overlapped transmit and receive region increases to $\pm 26.5^{\circ}$ in both directions and the energy is maintained

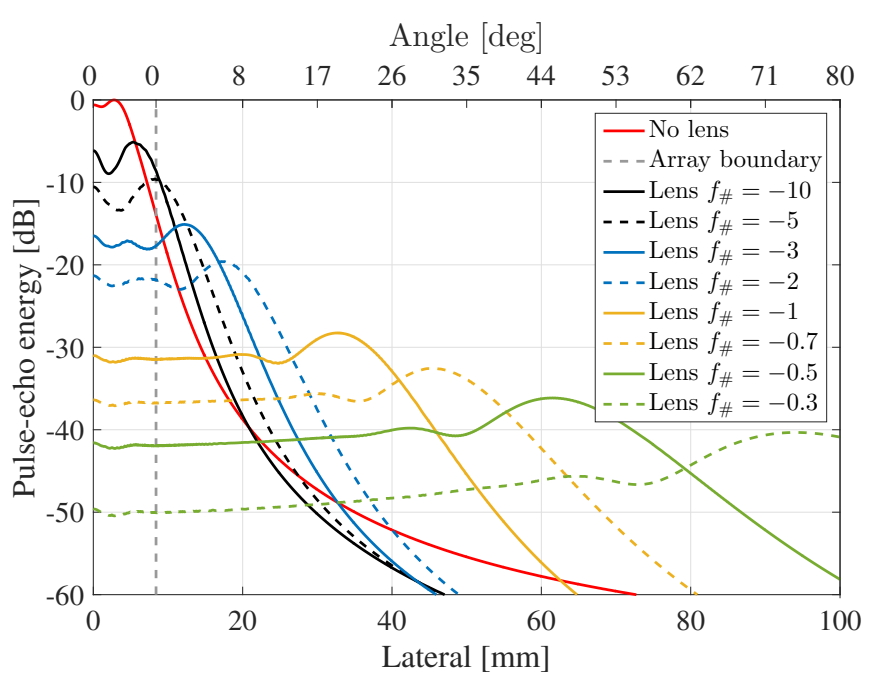

Fig. 3. Comparison of the pulse-echo energy for different lens f-numbers. The points are located on a line at $80 \mathrm{~mm}$ away from the surface of the transducer

within the FOV (yellow solid line in Fig. 3). By using all the elements in the transmit and placing the transmit focus in front of the array, this drop of the energy might be compensated partially.

Fig. 4 is illustrating three cross-planes (azimuth, elevation and C-plane) of a phantom with point targets simulated and beamformed with the proposed DAS beamforming method, with and without a diverging lens. The point targets are located along elevation dimension from $-30 \mathrm{~mm}$ to $30 \mathrm{~mm}$ in an axial range of $5 \mathrm{~mm}$ to $95 \mathrm{~mm}$. It can be seen from the figures that by using a diverging lens the FOV is extended compared to the flat RCA array.

To study the PSF characteristics as a function of lateral angle and radial distance, a point scatterer is imaged by sweeping it from $0^{\circ}$ to $40^{\circ}$ in lateral plane with steps of $10^{\circ}$ at radial distances from $10 \mathrm{~mm}$ to $60 \mathrm{~mm}$ from the center of the array. At each radial distance and lateral angle the fullwidth at half-maximum (FWHM) and cystic resolution (CR) values are calculated over a volume of $10 \mathrm{~mm} \times 10 \mathrm{~mm} \times 10 \mathrm{~mm}$ surrounding the point target. Fig. 5 is illustrating the measured FWHM and CR values as a function of depth and lateral angle. Moving away from the center of the elements towards the edges, the transmit wavefronts contact each other at a sharper point compared with the contact point at the center. This can be observed in Fig. 5 that, by moving away from the center towards the higher angular position in lateral plane, the elevation FWHM values become smaller while the CR values become larger. On the other hand, the lateral FWHM values stay constant, this is due to the intersection of the wavefronts in receive direction which is at the center of the receive elements and therefore the elevation FWHM values stay constant for all lateral angles.

\section{CONCLUSION}

In this paper, the imaging performance of a curved $62+62$ RCA 2-D array with a diverging lens, is quantitatively demon- 
no lens
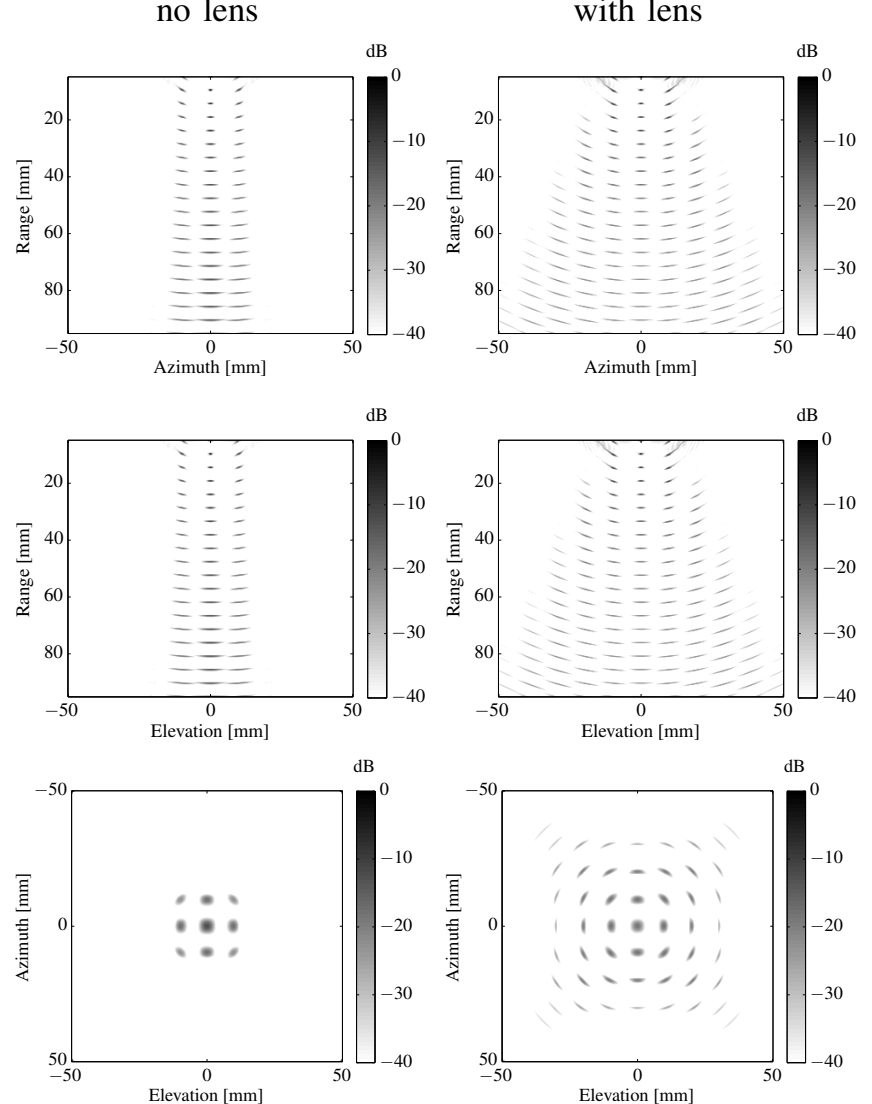

Fig. 4. Three cross-planes (azimuth, elevation, and C-plane) of a phantom with point scatterers imaged with and without a diverging lens $\left(f_{\#}=-1\right)$ normalized to their maximum values, are shown at a dynamic range of $40 \mathrm{~dB}$ The C-planes are at depth of $47 \mathrm{~mm}$.

strated. A SAI sequence with single element transmissions at a time, was designed for imaging down to $14 \mathrm{~cm}$ at a volume rate of $88 \mathrm{~Hz}$. The capabilities of a curved RCA 2-D array to effectively focus in both transmit and receive are investigated. A suitable DAS beamformer was introduced and implemented. Simulated results confirm that using a diverging lens can increase the imaging FOV and also that it is possible to perform dynamic transmit-receive focusing throughout the curvilinear FOV. Thereby, the inherent imaging limitation with flat RCA 2-D arrays, i.e., its forward looking rectilinear $\mathrm{FOV}$, is overcome by using a diverging lens. Overall, having a low channel count and a large FOV, offers the potential to fabricate arrays with large aperture sizes, which is important for abdominal scans. Thus by using a curved RCA 2-D array, 3 -D imaging with equipment in the price range of conventional 2-D imaging is possible.

\section{ACKNOWLEDGMENT}

This work was financially supported by grant 82-2014-4 from the Danish National Advanced Technology Foundation and from BK Ultrasound ApS, Herlev, Denmark.

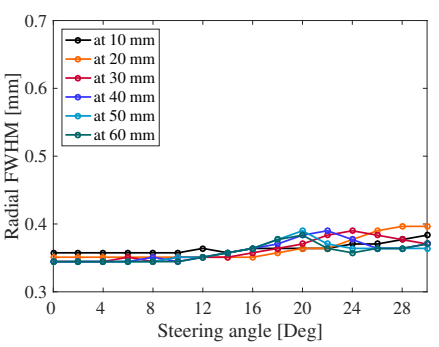

(a) Axial FWHM
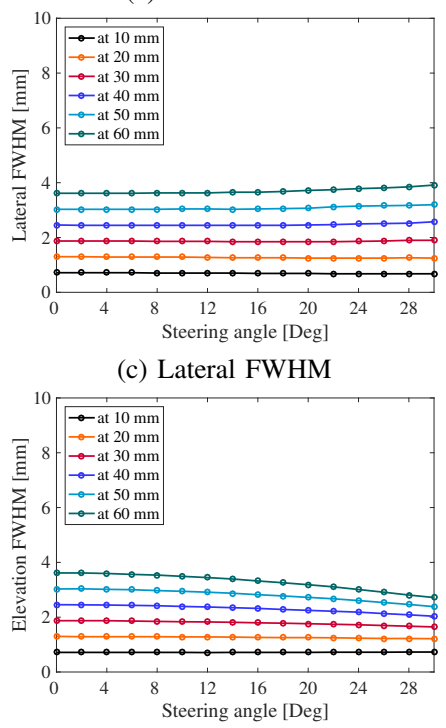

(e) Elevation FWHM (c) Lateral FWHM

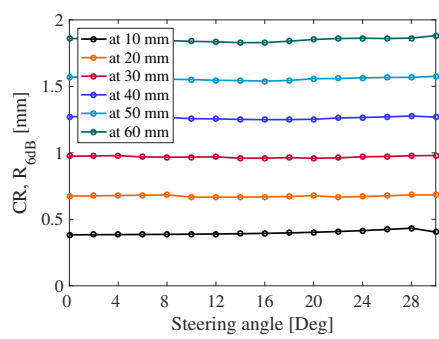

(b) CR $R_{6 d B}$

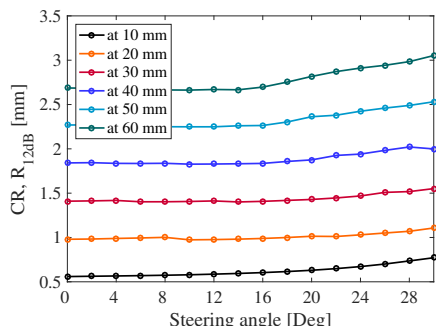

(d) CR $R_{12 d B}$

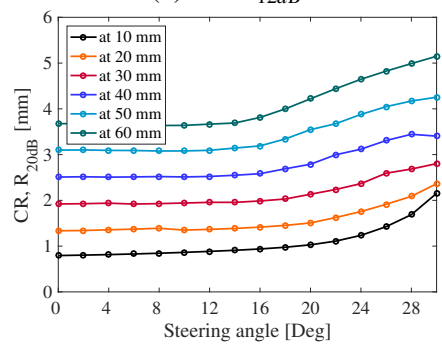

(f) CR $R_{20 d B}$
Fig. 5. CR and FWHM values calculated for point targets located at radial distances from $10 \mathrm{~mm}$ to $60 \mathrm{~mm}$ as a function of different azimuth steering angle away from the central forward-looking axis of the array.

\section{REFERENCES}

[1] C. E. M. Démoré, A. Joyce, K. Wall, and G. Lockwood, "Real-time volume imaging using a crossed electrode array," IEEE Trans. Ultrason. Ferroelec., Freq. Contr., vol. 56, no. 6, pp. 1252-1261, 2009.

[2] M. F. Rasmussen and J. A. Jensen, "3D ultrasound imaging performance of a row-column addressed 2D array transducer: a simulation study," in Proc. SPIE Med. Imag., 2013, pp. 1-11, 86750C.

[3] R. K. W. Chee, A. Sampaleanu, D. Rishi, and R. J. Zemp, "Top orthogonal to bottom electrode (TOBE) 2-D CMUT arrays for 3-D photoacoustic imaging," IEEE Trans. Ultrason., Ferroelec., Freq. Contr., vol. 61, no. 8, pp. 1393-1395, 2014.

[4] Phillips, "http://www.healthcare.philips.com,” February 2015.

[5] A. W. Joyce and G. R. Lockwood, "Crossed-array transducer for real-time 3D imaging," in Proc. IEEE Ultrason. Symp., 2014, pp. 2116-2120.

[6] J. A. Jensen and N. B. Svendsen, "Calculation of pressure fields from arbitrarily shaped, apodized, and excited ultrasound transducers," IEEE Trans. Ultrason., Ferroelec., Freq. Contr., vol. 39, pp. 262-267, 1992.

[7] J. A. Jensen, "Field: A program for simulating ultrasound systems," Med. Biol. Eng. Comp., vol. 10th Nordic-Baltic Conference on Biomedical Imaging, Vol. 4, Supplement 1, Part 1, pp. 351-353, 1996.

[8] C. Chang, K. Firouzi, K. K. Park, A. F. Sarioglu, A. Nikoozadeh, H. Yoon, S. Vaithilingam, T. Carver, and B. T. Khuri-Yakub, "Acoustic lens for capacitive micromachined ultrasonic transducers," Journal of Micromechanics and Microengineering, vol. 24, no. 8, p. 085007, 2014.

[9] M. F. Rasmussen, T. L. Christiansen, E. V. Thomsen, and J. A. Jensen, "3 D imaging using row-column-addressed arrays with integrated apodization - Part I: Apodization design and line element beamforming," IEEE Trans. Ultrason., Ferroelec., Freq. Contr., vol. 62, no. 5, pp. 947-958, 2015. 